\title{
Application of Differential Transform Method in Richards' Equation1
}

\author{
Zhong Xinran ${ }^{1, a}$, Dai Ying ${ }^{1, b, *}$, Chen $\mathrm{Xi}^{1, \mathrm{c}}$ \\ ${ }^{1}$ School of Aerospace Engineering and Applied Mechanics, Tongji University, Shanghai, China \\ a101839@tongji.edu.cn, bydai@tongji.edu.cn, c123cx@tongji.edu.cn
}

Keywords: differential transform method, Richards' equation, intermediate variable, approximate analytical solution.

Abstract. An approximate analytical solution of Richards' equation was derived by the differential transform method (DTM) in this paper. The basic idea is that the Richards' equation can be converted to an ordinary differential equation (ODE) by introducing the Boltzmann variable. Then DTM was applied to solve the resulting ODE. In the calculations, an intermediate variable was introduced in DTM for accelerating the convergence rate. In the end, an example was presented to demonstrate the accuracy of the present solution.

\section{Introduction}

The Richards' equation can be described as a sort of unsaturated single phase fluid flow in porous medium $^{[1-3]}$ (soil, concrete, etc). In most cases, Richards' equation is a nonlinear partial differential equation (PDE) about saturation of unsaturated porous media and can be written as ${ }^{[2]}$

$$
\frac{\partial S}{\partial t}=\frac{\partial}{\partial x}\left(D(S) \frac{\partial S}{\partial x}\right)
$$

where $S$ is the saturation of unsaturated porous media, $x$ and $t$ are the space and time coordinates, $D(S)$ is the hydraulic diffusivity function of the porous media.

Because the characteristics of materials and scope of application are different, several models such as Fredlund model ${ }^{[4]}$, Brooks-Corey model ${ }^{[5]}$, van Genuchten model ${ }^{[6]}$, etc, have been suggested for determining the basic parameter $D(S)$. Among them, the Brooks-Corey model is widely adopted for its simply description ${ }^{[7]}$.

The basic functional relationship between $D(S)$ and $S$ in Brooks-Corey model is commonly approximated by the power-law ${ }^{[5]}$

$$
D(S)=D_{0} S^{n},
$$

where $D_{0}$ and $n$ are the empirically-fitted constants ${ }^{[2]}$.

In the recent research, many approaches were adopted to solve the analytical solution of Richards' equation, for instance, the double integral technique ${ }^{[8]}$, traveling wave method (TWM ${ }^{[9]}$, DTM and homotopy perturbation method (HPM $)^{[7]}$.

In these approaches, double integral technique need a given wetting front as basic solution to search the approximate solution. TWM, which based on wetting front propagation, obtains the traveling wave approximate solution. DTM and HPM are based on polynomial expansion and they can get the series solution of Brooks-Corey model with power-law.

In this work, DTM is adopted to find an approximate analytical solution of the Richards' equation with $D(S)$ represented by the power-law and exponential-law, respectively.

\section{Initial and boundary conditions}

We consider the problem of the infiltration of water into a semi-finite layer of porous medium. According to Parlange's and Witelskis's work ${ }^{[1-3]}$, the initial condition can be described as a constant moisture concentration.

$$
\left.S\right|_{t=0}=0 \text {. }
$$

*Corresponding author: Dai Ying, School of Aerospace Engineering and Applied Mechanics, Tongji University, Shanghai, China

E-mail: ydai@tongji.edu.cn 
and the boundary conditions are given as ${ }^{[2]}$

$$
\left.S\right|_{x=0}=1,\left.S\right|_{x \rightarrow+\infty}=0 \text {. }
$$

As the boundary conditions describing, the water is saturated at the initial point where $x=0$ and it gradually infiltrates to the distal end $(S=0)$.

\section{DTM in Richards' equation}

The DTM was initially presented by Zhou ${ }^{[10]}$ in 1986, which uses Taylor series for the solution of linear or nonlinear differential equations in the form of polynomial. From the pioneer research on the $\mathrm{DTM}^{[11]}$, the basic conception of DTM is that each function $S(x)$ can be expressed as

$$
S(x)=\sum_{k=0}^{n} Y(k)\left(x-x_{0}\right)^{k},
$$

where $k$ is the derivation order and $Y(k)$ is the differential transformation of $S(x)$. The conversion relation between $Y(k)$ and $S(x)$ can be represented as

$$
Y(k)=\frac{1}{k !}\left[\frac{\mathrm{d}^{k} S(x)}{\mathrm{d} x^{k}}\right]_{x=x_{0}} .
$$

Most of time, the convergence rate of ordinary DTM is very slow while calculating the nonlinear differential equation ${ }^{[7]}$.To accelerating the convergence rate of the polynomial, we construct an intermediate variable $\xi=\xi(x, t)$, and let $\phi(x, t)=\phi(\xi)$. For Eq. 1, we introduce the Boltzmann variable $\left(\phi=x t^{-1 / 2}\right)^{[1]}$, then, Eq. 1 can be transformed into

$$
-\frac{1}{2} \phi \frac{\mathrm{d} S}{\mathrm{~d} \phi}=\frac{\mathrm{d}}{\mathrm{d} \phi}\left(D(S) \frac{\mathrm{d} S}{\mathrm{~d} \phi}\right) .
$$

The integration of Eq. $8^{[1,5]}$ is

$$
-2 D(S)=\frac{\mathrm{d} \phi}{\mathrm{d} S} \int_{0}^{S} \phi \mathrm{d} S .
$$

Substituting the Boltzmann variable $\phi$ into initial condition Eq. 3 and boundary conditions Eq. 4 , new boundary conditions can be rewritten as

$$
\left.\phi\right|_{S=1}=0,\left.\phi\right|_{S=0} \rightarrow+\infty \text {. }
$$

In this case, Eq. 1 with initial condition Eq. 3 and boundary conditions Eq. 4 are transformed into ordinary differential Eq. 8 with boundary conditions Eq. 9.

Then, we introduce an intermediate variable into DTM as follow

$$
\xi=\int_{1}^{S} \frac{D(S)}{S} \mathrm{~d} S
$$

which is the TWM special solution of Eq. 1 . The Boltzmann variable $\phi{ }^{[12]}$ can be expressed as

$$
\phi=\left\{\begin{array}{ll}
\sum_{k=0}^{+\infty} U(k)\left(\xi-\xi_{0}\right)^{k}, & \int_{1}^{0} \frac{D(S)}{S} \mathrm{~d} S<\xi \leq 0 \\
+\infty, & \xi=\int_{1}^{0} \frac{D(S)}{S} \mathrm{~d} S
\end{array},\right.
$$

where $\xi_{0}\left(=\left.\xi\right|_{S=1}\right)$ is the convergence center, and $U(k)$ is the differential transformation of $\phi(\xi)$

$$
U(k)=\left.\frac{1}{k !} \frac{\mathrm{d}^{k} \phi}{\mathrm{d} \xi^{k}}\right|_{\xi=\xi_{0}} .
$$

Introducing the intermediate variable $\xi$ into Eq. 8, we have

$$
-2 S=\frac{\mathrm{d} \phi}{\mathrm{d} \xi} \int_{0}^{S} \phi \mathrm{d} S
$$


13

According to theorems deduced from Eq. 5 and Eq. $6^{[11]}$, we obtain the transformed version of Eq.

$$
-2 W(k-1)=\sum_{i=0}^{k-1} Y_{1}(i) Y_{2}(k-1-i),
$$

where $W(k-1), Y_{1}(i)$ and $Y_{2}(k-1-i)(k=1,2,3, \ldots)$ are the differential transformation of $S, \frac{\mathrm{d} \phi}{\mathrm{d} \xi}$ and $\int_{0}^{S} \phi \mathrm{d} S$ respectively, and can be expressed as

$$
\begin{aligned}
& W(k-1)=\left.\frac{1}{(k-1) !} \frac{\mathrm{d}^{k-1} S}{\mathrm{~d} \xi^{k-1}}\right|_{\xi=\xi_{0}}, \\
& Y_{1}(i)=(i+1) U(i+1), \\
& Y_{2}(k-1-i)=\left.\frac{1}{(k-1-i) !} \frac{\mathrm{d}^{(k-1-i)}}{\mathrm{d} \xi^{(k-1-i)}}\left(\int_{0}^{S} \sum_{j=0}^{+\infty} U(j)\left(\xi-\xi_{0}\right)^{j} \mathrm{~d} S\right)\right|_{\xi=\xi_{0}},
\end{aligned}
$$

in which $Y_{1}(0)$ and $Y_{2}(0)$ are initial terms of $Y_{1}(i), Y_{2}(k-1-i)$ and can be shown as

$$
\begin{aligned}
& Y_{1}(0)=U(1), \\
& Y_{2}(0)=\int_{0}^{1} \sum_{j=0}^{+\infty} U(j)\left(\xi-\xi_{0}\right)^{j} \mathrm{~d} S .
\end{aligned}
$$

Considering the boundary condition Eq. 9 when $S=0$, we find that the case $\phi \rightarrow+\infty$ is satisfied naturally from Eq. 9. Substituted Eq. 10 and Eq. 11 into Eq. 9, we obtain

$$
U(0)=0 \text {. }
$$

Based on Eq. 14, and Eq. 18 to 20, we can obtain the analytical solution of Eq. 14 by calculating the parameters $W(k-1), Y_{1}(i)$ and $Y_{2}(k-1-i)$ in Eq. $15-$ Eq. 17 term by term.

\section{Numerical examples}

Example ${ }^{[3]}: D(S)=0.784287 S^{6}$. According to Eq. 10, we obtain

$$
\xi=0.784287 \frac{S^{6}-1}{6} \text {. }
$$

and the Boltzmann variable $\phi$ could be expressed as

$$
\phi=\left\{\begin{array}{ll}
\sum_{k=0}^{+\infty} U(k) \xi^{k} & ,-\frac{1}{6}<\xi \leq 0 \\
+\infty & , \xi=-\frac{1}{6}
\end{array} .\right.
$$

By choosing $k=1,2,3,4$ in Eq. 22, we can obtain $U(k)$ with each $k$, respectively. Substituting $U(k)$ into Eq. 22 and the 1-4 order approximation solutions are as follow

1 order approximation solution: $\phi=-4.225001 \xi$.

2 order approximation solution: $\phi=-4.397515 \xi-2.803513 \xi^{2}$.

3 order approximation solution: $\phi=-4.373594 \xi-2.788261 \xi^{2}-2.963912 \xi^{3}$

4 order approximation solution: $\phi=-4.373602 \xi-2.790588 \xi^{2}-2.981201 \xi^{3}-3.749841 \xi^{4}$.

The intermediate variable $\xi$ is given in Eq. 21.

Comparing the results with that obtained by finite element method (FEM), the results of Eq. 22 Eq. 26 are shown in Fig. 1. The results of saturation $S$ obtained from 4 order approximations Eq. 26 are compared with that from FEM in Table 1. 


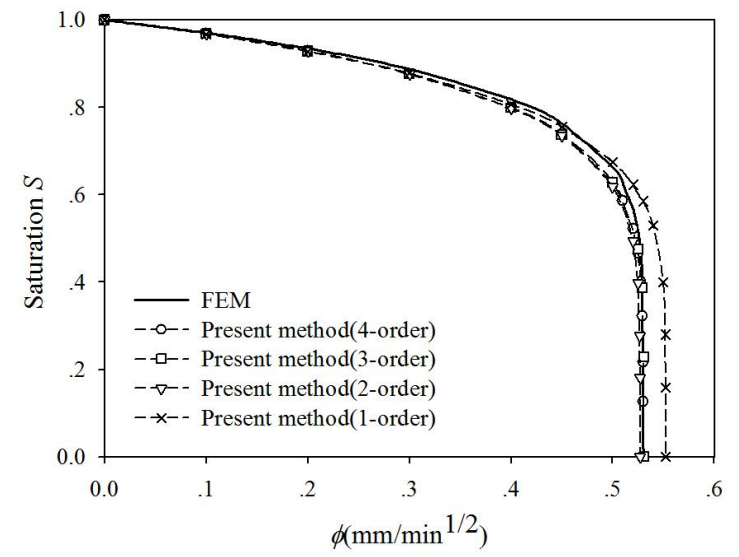

Fig. 1. Comparison of FEM and present method.

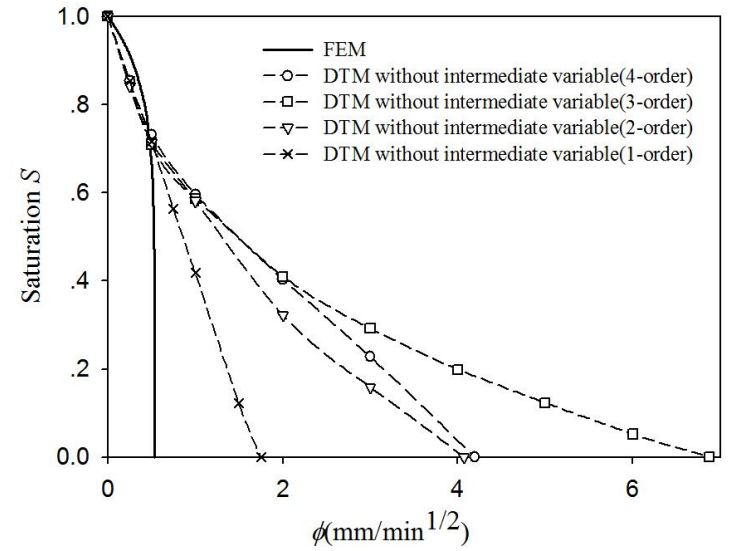

Fig. 2. Comparison of FEM and DTM without intermediate variable.

Table 1. Results of FEM and present method(4 order) of $\phi\left[\mathrm{mm} / \mathrm{min}^{1 / 2}\right]$.

\begin{tabular}{cccc}
\hline $\begin{array}{c}\text { Calculated distance } \\
\text { saturation } S\end{array}$ & FEM & $\begin{array}{c}\text { Present method } \\
\text { (4 order) }\end{array}$ & Relative error \\
\hline 1.00 & 0.0000 & 0.0000 & - \\
0.80 & 0.3986 & 0.4085 & $2.4839 \%$ \\
0.75 & 0.4413 & 0.4510 & $2.1968 \%$ \\
0.70 & 0.4716 & 0.4815 & $2.0978 \%$ \\
0.65 & 0.4927 & 0.5041 & $2.3131 \%$ \\
0.55 & 0.5164 & 0.5213 & $0.9537 \%$ \\
0.45 & 0.5259 & 0.5284 & $0.4759 \%$ \\
0.35 & 0.5291 & 0.5291 & $0.0147 \%$ \\
0.25 & 0.5299 & 0.5294 & $0.0953 \%$ \\
0.15 & 0.5300 & 0.5297 & $0.0641 \%$ \\
0.05 & 0.5301 & 0.5299 & $0.0204 \%$ \\
\hline
\end{tabular}

Meanwhile, adopting DTM without intermediate variable $\xi$, we obtain the 1-4 order approximate solutions of this example, which are compared with FEM in Fig. 2.

Compared with Fig. 1, the convergent speed of DTM without intermediate variable is so slow that makes it more difficult to converge to the FEM result.

\section{Conclusions}

In this paper, the DTM was adopted to solve the Richards' equation with Brooks-Corey model. Then an intermediate variable $\xi$ was introduced into DTM for the convergence acceleration. At last, an example was presented to demonstrate the accuracy of the presented approximate analytical solutions and the results were compared with that solved by FEM and DTM without intermediate variable. It is shown that the proposed method is robust and accurate.

\section{Acknowledgements}

This work was financially supported by the National Basic Research Program of China (973 Program: 2011CB013800).

\section{References}

[1] M. B. Parlance, S. N. Prasad, J. Y. Parlange: Extension of the Heaslet-Alksne technique to arbitrary soil water diffusivities. Water Resources Research 28 (1992), p.2793

[2] D. Lockington, J. Y. Parlange, P. Dux: Sorptivity and the estimation of water penetration into unsaturated concrete. Materials \& Structures 32 (1999), p. 342

[3] T. P. Witelski: Motion of wetting fronts moving into partially pre-wet soil. Advances in Water Resources 10 (2005), p. 1133 
[4] D. G. Fredlund: Equations for the soil-water characteristic curve. Canadian Geotechnical Journal 31 (1994), p.521

[5] R. H. Brooks, T. Corey: Hydraulic properties of porous media. Colorado State University: Hydrology Papers (1964), p. 27

[6] M. T. V. Genuchten: A closed-form equation for predicting the hydraulic conductivity of unsaturated soils. Soil Science Society of America Journal 44 (1980), p. 892

[7] M. Omidvar, A. Barari, M. Momeni: New class of solutions for water infiltration problems in unsaturated soils. Geomechanics \& Geoengineering An International Journal 5 (2010), p. 127

[8] D. G. Fredlund, H. Rahardjo, M. D. Fredlund: Theory of water flow through unsaturated soils (John Wiley \& Sons, New York 2012)

[9] T. P. Witelski: Perturbation analysis for wetting fronts in Richards' equation. Transport in Porous Media 27 (1997), p. 121

[10] J. K. Zhou: Differential transform and its applications for electrical circuits (Huazhong University Press, Wuhan 1986)

[11] C. K. Chen, S. H. Ho: Solving partial differential equations by two-dimensional differential transform method. Applied Mathematics \& Computation 106 (1999), p. 171

[12] R. R. Bruce, A. Klute, R. R. Bruce: The Measurement of Soil Moisture Diffusivity. Soil Science Society of America Journal 20 (1956), p. 458 